\title{
Modeling Floppy Iris Syndrome and the Impact of Phenylephrine on Iris Buckling
}

\author{
Nan $\mathrm{Qi}^{*}{ }^{\dagger}$, David Lockington ${ }^{\ddagger}$, Huiming Wang ${ }^{\S}$, \\ Nicholas A. Hill ${ }^{\dagger}$, Kanna Ramaesh ${ }^{\ddagger}$ and Xiaoyu Luo ${ }^{\dagger, \boldsymbol{\Phi}}$ \\ *Institute of Marine Science and Technology \\ Shandong University, Shandong 266237, P. R. China \\ ${ }^{\dagger}$ School of Mathematics and Statistics \\ University of Glasgow, UK \\ $\ddagger$ Tennent Institute of Ophthalmology \\ Gartnavel General Hospital, Glasgow, UK \\ $\S$ School of Civil Engineering \\ Xinjiang University, Xinjiang, P. R. China \\ 『Xiaoyu.luo@glasgow.ac.uk
}

Received 4 May 2018

Revised 19 May 2018

Accepted 20 May 2018

Published 11 July 2018

\begin{abstract}
Abnormal iris movement (floppy iris syndrome) during intraocular surgery is associated with an increased risk of intraoperative complications. We have previously investigated this scenario with respect to intracameral air in corneal endothelial transplantation, and described the concept of iris buckling. As a number of clinical interventions are recommended for addressing floppy iris syndrome, we wished to evaluate the impact of intracameral phenylephrine on iris buckling and so refine our mathematical model. We considered the stability of an iris structure under a uniform pressure loading. We performed mathematical and computational simulations to demonstrate iris buckling, and then altered the parameters to assess the impact of phenylephrine on the model. We elucidated a number of buckled iris configurations which become unstable as the intraocular pressure increased, for transversely isotropic iris material properties, and identified a positive correlation between the critical pressure and the iris stiffness. A mechanical analysis with a dilated pupil (mimicking phenylephrine use) was also conducted, and demonstrated a significant increase in the critical pressure required to induce iris buckling. We have shown that iris buckling can arise at lower pressures when the iris stiffness is reduced, as in floppy iris syndrome. The use of phenylephrine was shown to prevent iris movement (buckling) by increasing the required critical pressures. This refined model demonstrates the positive effectiveness of phenylephrine in the management of floppy iris syndrome and gives evidence to the clinical practice of using this as a preventative measure.
\end{abstract}

Keywords: Floppy iris syndrome; cataract surgery; intraocular surgery; phenylephrine; buckling stability.

This is an Open Access article published by World Scientific Publishing Company. It is distributed under the terms of the Creative Commons Attribution 4.0 (CC-BY) License. Further distribution of this work is permitted, provided the original work is properly cited. 


\section{Introduction}

Current strategies to improve the outcomes of cataract surgery focus on risk stratification by identifying any pre-existing conditions and peri-operative situations which could significantly increase the risk of intraoperative complications Day et al., 2015; Narendran et al., 2009; Sparrow et al., 2011]. One of these risk factors is abnormal intraoperative iris behavior, known as floppy iris syndrome. This condition was first described by Chang and Campbell in 2005 and consists of a triad of signs: poor pupillary dilation, iris billowing and subsequent iris prolapse through the corneal wounds Chang, 2008, 2009; Chang and Campbell, 2005; Chang et al., 2008a, 2008b; Neff et al., 2009]. This condition was originally described in association with previous systemic use of oral alpha blockers (such as Tamsulosin), but there are now an increasingly long list of associated medications Chang, 2008, 2009; Chang and Campbell, 2005; Chang et al., 2008a, 2008b; Neff et al., 2009].

There are a range of mechanical devices in use to address abnormal iris behavior, such as iris hooks or rings, but these are not necessary in all cases, and can result in localized iris sphincter trauma. While there are concerns regarding the accumulative effects of unlicensed intracameral adjuncts causing inflammation and toxic anterior segment syndrome Lockington et al., 2010], it has been our clinical experience and common practice that the use of intracameral phenylephrine can both dilate and stiffen the floppy iris, and so result in a reduction and normalization of the iris movement arising from the intraoperative fluidics Carifi and Kopsachilis, 2013; Gurbaxani and Packard, 2007; Lockington and Gavin, 2009; Lorente et al., 2012; Manvikar and Allen, 2006; Nguven et al., 2007. The relationship between phenylephrine and porcine pupil diameter has been quantified ex vivo in Whitcomb et al. 2009] and found to cause an increase of modulus in the iris tissue by approximately a factor of two.

In our previous work, normal iris behavior was modeled in the setting of an air bubble in the anterior chamber during endothelial graft surgery [Lockington et al., 2012]. We hypothesized that the reason for pupil block/angle closure which can arise following such surgery was due to iris buckling, and proceeded to develop a three-dimensional elastic isotropic model to demonstrate this scenario. In that study, we accounted for the isotropic material properties and predicted deformation of the iris under pressure. Our model was solved using finite element method and used to investigate the critical intraocular pressure (IOP) required to cause the iris buckling. However, the iris material properties were assumed to be oversimplified and we did not go on to evaluate the impact of the intrinsic iris properties on the model for other types of intraocular surgery (of which cataract surgery is the most common), or any potential clinical solutions to this situation.

Developing a detailed mathematical model of mechanical behavior of the normal iris and understanding the subsequent impact from floppy iris syndrome or intracameral pharmacological agents can help guide the clinician towards effective management of this intraoperative scenario. In this current paper, we wish to propose a 
refined mathematical model of the human iris as an orthotropic elastic material. We then use this updated model to study the effect of floppy iris syndrome by looking at the stability of the normal and abnormal iris structure under pressure loading. We also evaluate the impact of phenylephrine in a clinical scenario.

\section{Methods}

\subsection{Geometry and reference configuration}

We modeled the iris as an axisymmetric annular disc with a central aperture (mimicking the pupil). The lens is a fixed posterior structure and so is presented as an analytical rigid body below the iris plate. The external edge of the iris tissue is also fixed peripherally to the ciliary body and sclera. Based on the published averaged human data Amini and Barocas, 2010; Leung et al., 2010], we assumed that the iris has a uniform thickness of $0.34 \mathrm{~mm}$ with a hemispherical tip, the anterior chamber width is $12.37 \mathrm{~mm}$ and the lens diameter is $9.0 \mathrm{~mm}$, see in Fig. 1 The iris has no concavity and the iridolenticular contact distance is $0.77 \mathrm{~mm}$ Liebmann et al., 1995]. The iris-zonule distance is $0.69 \mathrm{~mm}$. The pupil diameter, based on porcine iris tissue, is between $5 \mathrm{~mm}$ to $6.2 \mathrm{~mm}$ after pharmacological pupil dilatation [Whitcomb et al., 2009].

(a)

(b)
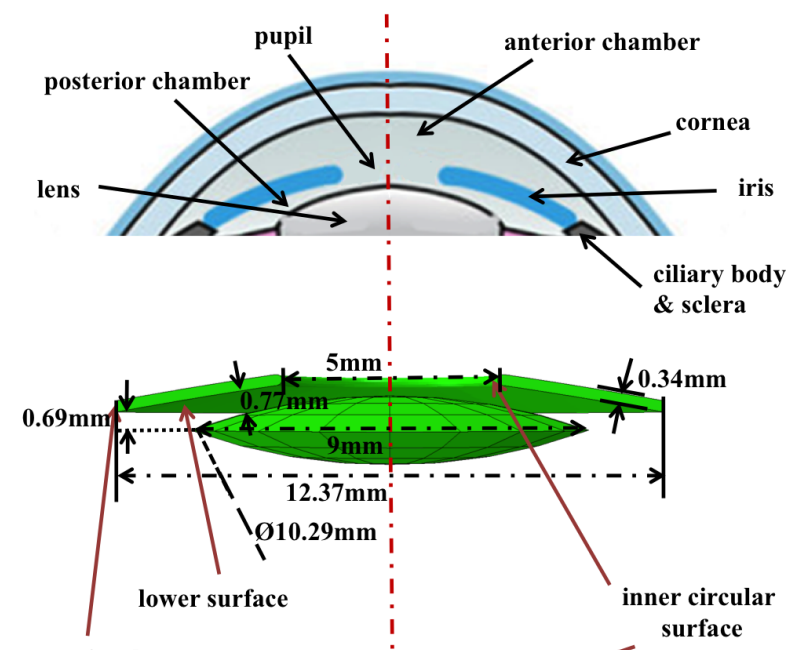

(c)

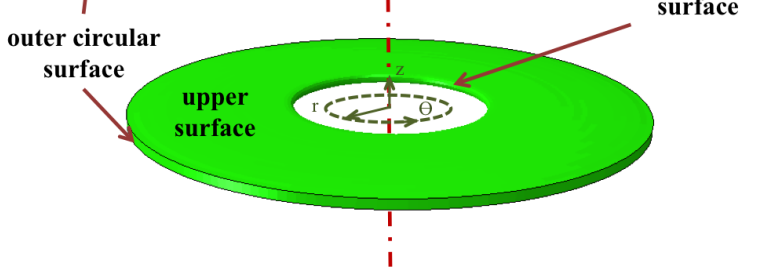

Fig. 1. (a) The side view of the eye structure; (b) the iris structure with the geometric information, and (c) the three-dimensional model of the iris, its relation with (b) is indicated. 


\subsection{Linear bifurcation analysis}

We defined the reference configuration described by a cylindrical coordinate system $(r, \theta, z)$, and assumed that the iris behaves as an elastic material occupying volume $V$ with boundary $\Gamma$. We then assumed that all free surfaces of the iris are subject to a constant IOP of $P$. In the absence of an external body force, the total excess potential energy of the iris structure is

$$
\Pi=\frac{1}{2} \int_{V} \varepsilon^{T} \mathbf{C} \varepsilon d V-\int_{\Gamma} \mathbf{u}^{T} P \mathbf{n} d S,
$$

where $\mathbf{C}$ is the fourth-order elasticity tensor (the tensor form is discussed below), $\mathbf{n}$ is the unit normal vector at the boundary $\Gamma$, and $\varepsilon$ is the finite strain tensor.

Using the principle of virtual displacement method, we obtained the equilibrium equations from Eq. (1). With the finite element discretization, i.e. using the nodal displacement vector $\mathbf{U}$ to represent $\mathbf{u}$ and $\boldsymbol{\varepsilon}$, we can express the system in the matrix form

$$
\mathbf{K U}=\mathbf{R} \text {, }
$$

where $\mathbf{K}$ is the stiffness matrix and $\mathbf{R}$ is the discretized load vector (for details see in Bathe's book [Bathe, 2006]).

The iris tissue was modeled as an orthotropic material, and $\mathbf{C}$ has nine independent parameters and can be expressed in cylindrical coordinates as

$$
\mathbf{C}^{-1}=\left(\begin{array}{cccccc}
\frac{1}{E_{r}} & -\frac{\nu_{r \theta}}{E_{\theta}} & -\frac{\nu_{r z}}{E_{z}} & 0 & 0 & 0 \\
-\frac{\nu_{\theta r}}{E_{r}} & \frac{1}{E_{\theta}} & -\frac{\nu_{\theta z}}{E_{z}} & 0 & 0 & 0 \\
-\frac{\nu_{z r}}{E_{r}} & -\frac{\nu_{z \theta}}{E_{\theta}} & \frac{1}{E_{z}} & 0 & 0 & 0 \\
0 & 0 & 0 & \frac{1}{G_{\theta z}} & 0 & 0 \\
0 & 0 & 0 & 0 & \frac{1}{G_{z r}} & 0 \\
0 & 0 & 0 & 0 & 0 & \frac{1}{G_{r \theta}}
\end{array}\right),
$$

where $E_{i}(i=r, \theta, z)$ are the elastic moduli in the three mutually orthogonal directions, $\nu_{i j}(i, j=r, \theta, z)$ represent the Poisson's ratios, $\nu_{i j} / E_{i}=\nu_{j i} / E_{j}$, and $G_{i j}$ are the corresponding shear moduli [Timoshenko and Gere, 1961].

We used linear buckling analysis to assess the stability characteristics of the iris structure Wittrick and Williams, 1973]. It involved finding the critical pressure load for which the stiffness matrix $\mathbf{K}$ becomes singular in the following eigenvalue problem. An incremental loading pattern, $P=P^{\text {base }}+\lambda_{k} P^{\text {inc }}$, is defined, where $\lambda_{k}$ is the scaling factor, which becomes the eigenvalue when the critical conditions 
are reached

$$
\left(\mathbf{K}_{0}^{\mathrm{cr}}+\lambda_{k} \mathbf{K}_{\Delta}^{\mathrm{cr}}\right) \mathbf{U}_{k}^{\mathrm{cr}}=\mathbf{0},
$$

where $\mathbf{K}_{0}^{\text {cr }}$ is the stiffness matrix corresponding to the base state, $\mathbf{K}_{\Delta}^{\mathrm{cr}}$ is due to the incremental loading, and $\mathbf{U}_{k}^{c r}$ are the eigenvectors of the $k$ th buckling mode Bathe, 2006; Systémes, 2013.

Following a grid independence test by refining mesh size sequentially, we constructed a finite element mesh with 19,610 nodes and 14,245 hexahedral elements. The finite element model was implemented using the commercial package ABAQUS 6.13 (SIMULIA, Providence, RI).

\subsection{Elastic properties}

The iris, like many biological tissues, was assumed to be nearly incompressible with Poisson's ratio equal to 0.499 [Heys and Barocas, 1999; Miller, 2005].

Based on the tests of porcine iris tissue, the iris material has its azimuthal elastic modulus $E_{\theta}$ to be $2.97 \mathrm{kPa}$, and the radial elastic modulus $E_{r}$ to be $4.00 \mathrm{kPa}$, presenting a clearly orthotropic behavior Whitcomb et al., 2009]. The longitudinal modulus $E_{z}$ is assumed to be the same as $E_{r}$, and $G_{r z}=G_{\theta z}=G_{r \theta}=E_{r} / 2(1+$ $\left.\nu_{r z}\right)$. Simulating the clinical scenario of exposure to intracameral phenylephrine, the radial moduli becomes $6.9 \mathrm{kPa}$.

The typical IOP in humans is around $10-20 \mathrm{mmHg}(1 \mathrm{mmHg} \approx 0.13 \mathrm{kPa})$ in normal subjects [Lockington et al., 2012; Heijl et al., 2002] but can exceed $50 \mathrm{mmHg}$ due to intraocular fluidics [Lockington et al., 2012]. To mimic the increase in IOP during intraocular surgery, we applied a pressure loading incrementally from $P^{\text {base }}=10.00 \mathrm{mmHg}$ onwards. The loading was applied simultaneously to the upper, lower and inner circular surfaces of the iris as indicated in Fig. 1(c). The displacements of the outer circular surface were fully constrained to mimic the anchoring by the ciliary body. Surface tension and shear forces due to aqueous flow were considered to be negligible.

\section{Results}

\subsection{Buckling analysis for orthotropic control case}

We conducted the stability analysis of the normal iris under loading. The iris was modeled as a homogeneous orthotropic material with an azimuthal elastic modulus of $2.97 \mathrm{kPa}$, and a radial elastic modulus of $4.00 \mathrm{kPa}$. We chose $P^{\text {base }}=P^{\text {inc }}=$ $10.00 \mathrm{mmHg}$ and increased the IOP (i.e., increased the scale factor $\lambda_{k}$ ) until the critical condition was reached.

The critical load $P_{c}$ when the iris first buckles was found to be $17.42 \mathrm{mmHg}$ for the chosen elastic moduli. With further increases in IOP, higher buckling modes also occurred. We labeled each buckling mode according to the number of local maxima across the structure, for example, mode $n=2$ has 2 maxima as shown in Fig. 2(c). 


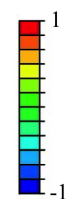

(a)

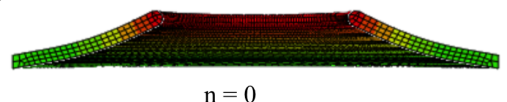

$\mathrm{P}_{\mathrm{c}}=17.42 \mathrm{mmHg}$

(c)

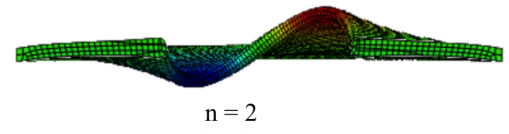

(e)

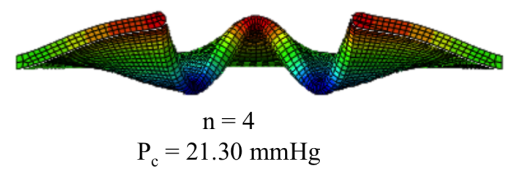

(b)

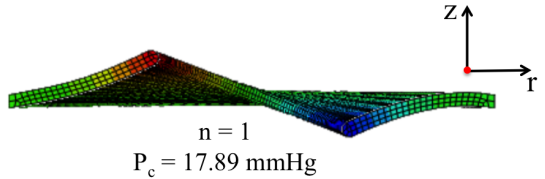

(d)

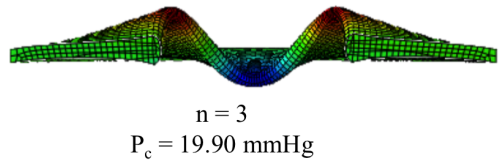

(f)

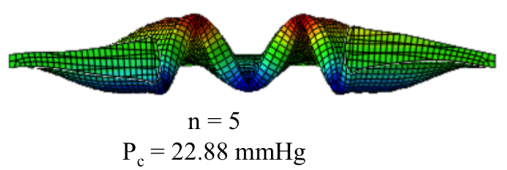

Fig. 2. The first six buckling modes in the $r \theta$-plane view, in which $E_{r}=E_{z}=4.00 \mathrm{kPa}, E_{\theta}=$ $2.97 \mathrm{kPa}$. The color indicates the mode's longitudinal displacement. Note that the magnitude is unified from eigenvalue analysis.

The $n=0$ mode is axisymmetric while for $n>0$, mode $n$ has $n$ symmetries. As in the analytical solution [Bloom and Coffin, 2000], the annular plate always buckles into the axisymmetric form regardless of the hole size. The subsequent increase in fluidics behind the iris will exacerbate the buckling shapes/tissue instability, eventually leading to prolapse of tissue through the incision wounds during cataract surgery.

\subsection{Buckling analysis for phenylephrine-activated iris tissue}

We have previously noted that the elastic properties/stiffness of the iris can be very different in abnormal clinical situations: for example, in floppy iris syndrome or a traumatized tonic pupil, the elastic moduli is significantly decreased, and conversely, the introduction of intracameral phenylephrine has been shown to have a positive clinical effect by stiffening the iris (modulus). We wondered if this mechanical effect combined with iris dilatation could increase the critical pressure, and hence, reduce the potential for the iris buckling.

To further investigate the dilated pupil diameter and iris stiffness variance due to intracameral phenylephrine use, we repeated the analysis with different dilated pupil sizes and material stiffness. We changed the elastic moduli in the orthotropic iris model using a proportion factor $\eta$, so that the elastic moduli becomes $\eta E_{i}$, $(i=r, \theta, z)$. With $\eta<1$, the iris becomes soft (floppy), while with $\eta>1$, the iris is stiffened by phenylephrine.

The relationship between the critical pressures and $\eta$ is summarized in Table 1 highlighting how a uniform augmentation/reduction in stiffness can increase/reduce the critical pressures. In addition, the differences in critical pressure between the 
Table 1. The critical pressures $(\mathrm{mmHg})$ for the first six modes with different material stiffness factor $\eta$. The bold values are the critical pressures for the first buckling mode.

\begin{tabular}{ccccccc}
\hline$\eta$ & \multicolumn{5}{c}{$P_{c}[\mathrm{mmHg}]$} \\
\cline { 2 - 6 } & $n=0$ & $n=1$ & $n=2$ & $n=3$ & $n=4$ & $n=5$ \\
\hline 0.6 & $\mathbf{1 6 . 5 4}$ & 17.28 & $/$ & $/$ & $/$ & $/$ \\
0.7 & $\mathbf{1 6 . 8 2}$ & 17.47 & 18.38 & 19.28 & $/$ & $/$ \\
0.8 & $\mathbf{1 7 . 0 4}$ & 17.62 & 18.49 & 19.46 & 20.60 & $/$ \\
0.9 & $\mathbf{1 7 . 2 0}$ & 17.73 & 18.61 & 19.66 & 20.93 & 22.36 \\
1 & $\mathbf{1 7 . 4 2}$ & 17.89 & 18.76 & 19.90 & 21.30 & 22.88 \\
1.5 & $\mathbf{1 9 . 5 2}$ & 19.54 & 20.40 & 22.06 & 24.19 & 26.53 \\
2 & 24.61 & $\mathbf{2 3 . 6 1}$ & 24.07 & 26.20 & 28.99 & 31.91 \\
4 & 72.21 & 59.13 & $\mathbf{5 0 . 1 3}$ & 50.24 & 53.03 & 56.62 \\
\hline
\end{tabular}

\section{The relationship of first critical pressure and material stiffness factor}

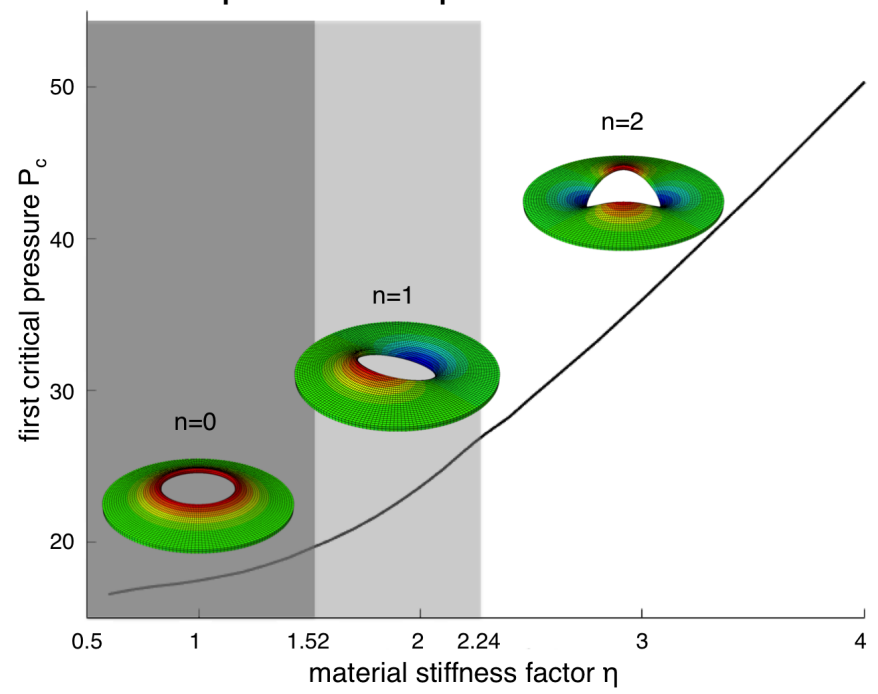

Fig. 3. The plot of first critical pressures with respect to the material stiffness factor $\eta$. In the dark gray region, the iris first buckles into mode 0, while it buckles into mode 1 in the light gray region and mode 2 in the white region.

individual modes become greater with the increased material stiffness except for the behaviors of mode 0 which are more sparse. We noticed that as $\eta$ reduces, the structure became very soft and yielded large deformation. As the result, it is harder to obtain converged solutions when loading is high. We also noted from Fig. 3 that a stiffer material is more likely to lose stability to higher buckling modes first. For example, the iris becomes unstable at mode 1 when $\eta \geq 1.52$ at the critical pressure of $19.65 \mathrm{mmHg}$, instead of mode 0 in the softer cases; at $\eta>2.24$, the iris becomes unstable to mode $n=2$ at a critical pressure of $P_{c}=26.50 \mathrm{mmHg}$.

Table 2 shows the critical pressure $P_{c}$ for the first six buckling modes with different dilated pupil diameter $d_{p}$ and the corresponding iris thickness $t_{i}$. Note 
Table 2. Values of $P_{c}, d_{p}, t_{i}$ for the first six buckling modes. The bold values are the critical pressures of the first buckling.

\begin{tabular}{lccccccc}
\hline$d_{p}[\mathrm{~mm}]$ & $t_{i}[\mathrm{~mm}]$ & \multicolumn{7}{c}{$P_{c}[\mathrm{mmHg}]$} \\
\cline { 3 - 8 } & & $n=0$ & $n=1$ & $n=2$ & $n=3$ & $n=4$ & $n=5$ \\
\hline 5.00 & 0.3400 & $\mathbf{1 7 . 4 2}$ & 17.89 & 18.76 & 19.90 & 21.30 & 22.88 \\
5.50 & 0.3488 & $\mathbf{1 8 . 3 1}$ & 18.62 & 19.29 & 20.19 & 21.45 & 22.91 \\
6.00 & 0.3583 & $\mathbf{1 9 . 9 9}$ & 20.06 & 20.47 & 21.20 & 22.20 & 23.45 \\
6.50 & 0.3686 & 22.02 & $\mathbf{2 1 . 8 1}$ & 21.85 & 22.34 & 23.17 & 24.27 \\
\hline
\end{tabular}

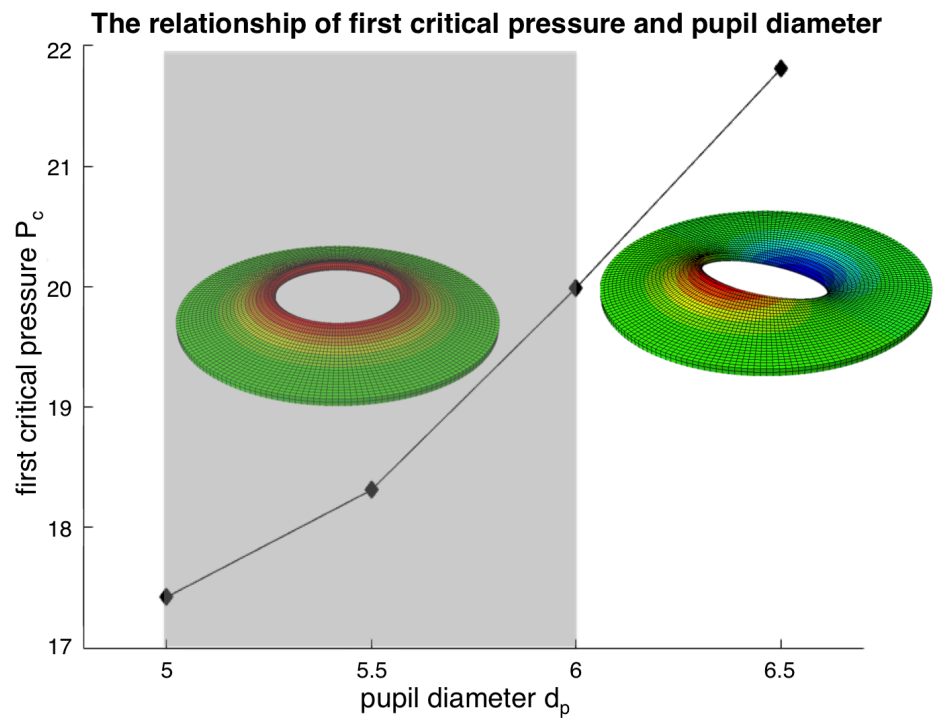

Fig. 4. The plot of the first critical pressure with respect to the degree of dilation/pupil size. In the dark gray region, the iris first buckles into mode 0 , while it buckles into mode 1 in the light gray region.

that the addition of phenylephrine causes the pupil to dilate, and since the iris volume is conserved, the iris thickness has to increase.

We note the positive correlation between the buckling pressures and the dilated pupil length in Table 2] a large/dilated pupil hole increases the critical pressure.

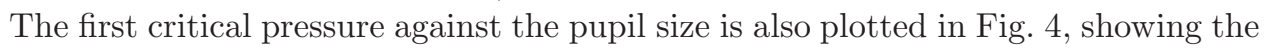
more dilated the pupil, the higher the buckling modes that become unstable first.

The critical pressure at different degrees of the pupil dilation is shown in Fig. 5 . Clearly, dilatation increases the critical pressure for all buckling modes, and therefore stabilize the system. This is because dilation reduces the surface area and thus the iris thickens. This in turn, increases the critical buckling pressure.

The critical IOP required for each buckling mode is significantly lowered when the material becomes less stiff (floppy), as might be expected from other studies of buckling in elastic tissue Han, 2007; Townsend et al., 1975]. However, the use of intracameral phenylephrine has a synergistic effect to minimize intraoperative iris 


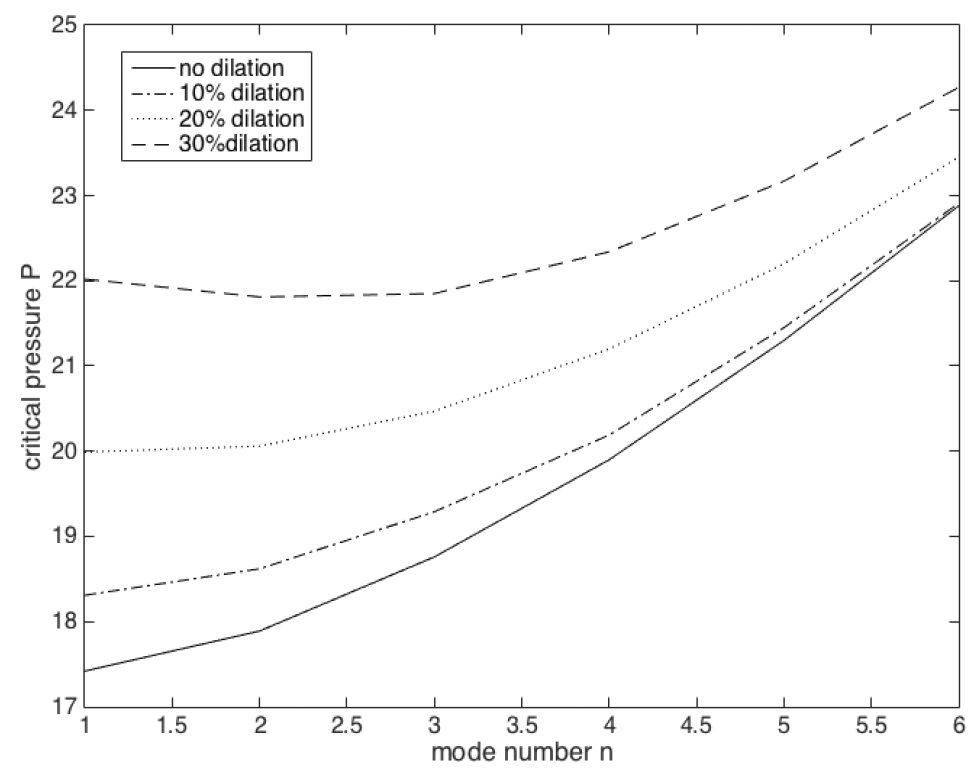

Fig. 5. The critical pressures for different degree of dilations of the pupil.

buckling by (a) increasing the material stiffness moduli and (b) dilating the pupil aperture. We note that these two factors are highly correlated, as is demonstrated in Figs. 3 and 4.

\section{Discussion}

This study aimed to study abnormal iris movement during intra-ocular surgery using a finite element mechanical model of iris. Our 3D finite element model showed that the iris tissue instability found during the surgery is due to iris buckling. The reason for the buckling is, because the outer ring of the iris is clamped, although the pressure is applied to all sides, the pressure on the top and bottom surfaces do not produce much net effect, and the pressure on the inner surface of the iris is responsible for the buckling. This is different to placing a round disk of finite thickness with a circular hole in the bottom of a swimming pool; since the disk is not clamped on any side, it will not buckle even if the pressure is huge. We also identified the key parameters in iris buckling, the IOP loading and the impact of floppy iris behavior.

We demonstrated that phenylephrine is effective in reducing the movement of the iris during cataract surgery. It is worth mentioning that the effects of phenylephrine have been debated in literature. Some argued that there is no or reduced dilatation caused by phenylephrine [Gurbaxani and Packard, 2015], while others stated the opposite [Vazquez-Ferreiro et al., 2017; Lorente et al., 2012; Lundqvist et al., 2014]. Since the injection of phenylephrine is used frequently to dilate small pupils in 
cataract surgery, and phenylephrine eye drops are used in all clinics to dilate pupils, we believe it is important to study the impact of the phenylephrine. In addition, the iris tissue of patients (i.e. stiffness) may be different compared to a normal iris, and without the patient-specific iris data, we performed a parameter study looking at the effects of a range of stiffness changes. Therefore, despite the simplifications, our finding is qualitatively representative of the clinical situations, and our simulations are visually consistent with the intra-operative surgical findings.

The majority of eye modeling studies have focused on ocular fluid mechanics [Canning et al., 2002; Fitt and Gonzalez, 2006; Fleck, 1990], or simplified fluidstructure interaction [Huang and Barocas, 2004, 2006]; some of the studies have considered the iris and its interaction with aqueous humour Canning et al., 2002; Fitt and Gonzalez, 2006; Heys et al., 2001]. However, to the best of our knowledge, three-dimensional modeling of iris buckling under pressure loading accounting for the anisotropy of the iris tissue has not been reported. Developing a detailed mathematical model of mechanical behavior of the normal iris and understanding the subsequent impact of floppy iris syndrome can help guide the clinician to the effective management of this intraoperative complication. Compared to our previous study based on a simplified mathematical model [Lockington et al., 2012], this work provides a quantitative measure for structure-based iris buckling with realistic material properties for the first time. We have shown that the critical pressure of buckling is dependent on both the iris stiffness and pupil size. We also have demonstrated how intracameral phenylephrine can reduce the instability of the iris buckling. We remark that the effect of phenylephrine to iris buckling is similar to the effect of arterial contraction and dilatation to its critical buckling pressure, i.e., higher wall thickness to radius ratio increases the critical pressure Han, 2007; Havman et al., 2013. We believe our model of iris buckling is the first step towards understanding and preventing this complication.

So, what does this mean for the clinician? The majority of cataract surgeons will have encountered floppy iris syndrome, and we trust our computer simulation and cross-sectional diagrams are an accurate representation of this clinical scenario. We believe our model demonstrates that floppy iris syndrome occurs due to reduced intrinsic iris stiffness properties, and that iris buckling can be induced at lower pressures/fluidics. As the clinical scenario appears to be minimized through stiffening of the iris with phenylephrine, it would be our recommendation that intracameral phenylephrine is used prior to the initiation of iris buckling, to minimize the adverse consequences. In other words, any buckling mode is unwelcome to the surgeon, as it means the iris has started to display abnormal movement behavior. This is a situation we all wish to avoid, as it can lead to iris prolapse and further complications, so we suggest the use of intracameral phenylephrine as the first preventative step.

One of the limitations of this study is the lack of specific material iris properties, which can change due to trauma or pharmacological intervention during eye surgery, and have different qualities dependent on their inherent physiological and/or pathological conditions. Since the mechanical properties of specific human 
iris tissue were not available, we had to estimate our model parameters based on measurements of animal (canine and porcine) iris which were varied [Heys and Barocas, 1999; Whitcomb et al., 2009]. Therefore, although our model predictions may explain the mechanism in general, it remains a challenge to provide a patientspecific prediction. In addition, our model is based on simplifications of the complex architecture of the iris and the anterior eye. In particular, the active contraction of the iris sphincter and dilator smooth muscles were ignored. Activation of iris smooth muscles can alter the iris contour and mechanical response and could therefore change the results of current model. In addition, the inhomogeneity of the iris structure may also alter the outcome. However, while these factors may change the values of the critical pressures predicted, we expect our qualitative findings will not change.

\section{Conclusion}

We used a finite element orthotropic iris mechanical model to understand the potential iris movements due to fluidics during intra-ocular surgery. The model predicts the critical pressure under which the iris may lose its stability. The situation is exacerbated by the degree of floppiness of the iris since the critical pressure is lower when the iris is less stiff. We suggest that iris buckling can arise at lower pressures when the iris stiffness is reduced, as in floppy iris syndrome. This model demonstrates the positive impact of phenylephrine in the management of floppy iris syndrome and gives evidence to the clinical practice of using this as a preventative measure.

\section{Acknowledgments}

This project is supported by the EPSRC grant (EP/I029990/1), Dr. Nan Qi would like to thank "The Fundamental Research Funds" of Shandong University, China, and Dr. Li Cai is supported by the National Natural Science Foundation of China (No. 11471261). We are also grateful to Dr. Peter Stewart and Prof. Ray Ogden for their helpful suggestions.

\section{References}

Amini, R. and Barocas, V. H. [2010] "Reverse pupillary block slows iris contour recovery from corneoscleral indentation," Journal of Biomechanical Engineering 132(7), 071010 .

Bathe, K.-J. [2006] Finite Element Procedures (Klaus-Jurgen Bathe).

Bloom, F. and Coffin, D. [2000] Handbook of Thin Plate Buckling and Postbuckling (Chapman and Hall, CRC Press).

Canning, C. R., Greaney, M. J., Dewynne, J. N. and Fitt, A. D. [2002] "Fluid flow in the anterior chamber of a human eye," Mathematical Medicine and Biology 19(1), $31-60$. 
Carifi, G. and Kopsachilis, N. [2013] "Alpha-1 adrenergic agonists for the prevention of floppy iris syndrome," Ophthalmology 120(7), e43.

Chang, D. F. [2008] "Use of malyugin pupil expansion device for intraoperative floppyiris syndrome: Results in 30 consecutive cases," Journal of Cataract and Refractive Surgery 34(5), 835-841.

Chang, D. F. [2009] "Why bph treatment can complicate cataract surgery," Ophthalmology News.

Chang, D. F. et al. [2008a] "Ascrs white paper: Clinical review of intraoperative floppy-iris syndrome," Journal of Cataract and Refractive Surgery 34(12), 2153-2162.

Chang, D. F. et al. [2008b] "Clinical experience with intraoperative floppy-iris syndrome: Results of the 2008 ascrs member survey," Journal of Cataract and Refractive Surgery 34(7), 1201-1209.

Chang, D. F. and Campbell, J. R. [2005] "Intraoperative floppy iris syndrome associated with tamsulosin," Journal of Cataract and Refractive Surgery 31(4), 664-673.

Day, A., Donachie, P., Sparrow, J. and Johnston, R. [2015] "The royal college of ophthalmologists' national ophthalmology database study of cataract surgery: Report 1, visual outcomes and complications," Eye 29(4), 552-560.

Fitt, A. D. and Gonzalez, G. [2006] "Fluid mechanics of the human eye: Aqueous humour flow in the anterior chamber," Bulletin of Mathematical Biology 68(1), 53-71.

Fleck, B. W. [1990] "How large must an iridotomy be?" British Journal of Ophthalmology 74(10), 583-588.

Gurbaxani, A. and Packard, R. [2007] "Intracameral phenylephrine to prevent floppy iris syndrome during cataract surgery in patients on tamsulosin" Eye 21, 331-332.

Han, H. C. [2007] "A biomechanical model of artery buckling," Journal of Biomechanics 40(16), 3672-3678.

Hayman, D. M., Zhang, J., Liu, Q., Xiao, Y. and Han, H. C. [2013] "Smooth muscle cell contraction increases the critical buckling pressure of arteries," Journal of Biomechanics 46(4), 841-844.

Heijl, A., Leske, M. C., Bengtsson, B., Hyman, L., Bengtsson, B. and Hussein, M. [2002] "Reduction of intraocular pressure and glaucoma progression: Results from the early manifest glaucoma trial," Archives of Ophthalmology 120(10), 1268-1279.

Heys, J. J. and Barocas, V. H. [1999] "Mechanical characterization of the bovine iris," Journal of Biomechanics 32(9), 999-1003.

Heys, J. J., Barocas, V. H. and Taravella, M. J. [2001] "Modeling passive mechanical interaction between aqueous humor and iris," Journal of Biomechanical Engineering 123(6), 540-547.

Huang, E. C. and Barocas, V. H. [2004] "Active iris mechanics and pupillary block: Steadystate analysis and comparison with anatomical risk factors," Annals of Biomedical Engineering 32(9), 1276-1285.

Huang, E. C. and Barocas, V. H. [2006] "Accommodative microfluctuations and iris contour," Journal of Vision 6(5), 10.

Leung, C. K. et al. [2010] "Comparisons of anterior segment biometry between chinese and caucasians using anterior segment optical coherence tomography," British Journal of Ophthalmology 94(9), 1184-1189.

Liebmann, J. M., Tello, C., Chew, S.-J., Cohen, H. and Ritch, R. [1995] "Prevention of blinking alters iris configuration in pigment dispersion syndrome and in normal eyes," Ophthalmology 102(3), 446-455.

Lockington, D. and Gavin, M. P. [2009] "Intraoperative floppy-iris syndrome: Role of the bimanual approach," Journal of Cataract and Refractive Surgery 35(6), 964. 
Lockington, D., Luo, X., Wang, H., Hill, N. A. and Ramaesh, K. [2012] "Mathematical and computer simulation modelling of intracameral forces causing pupil block due to air bubble use in descemet's stripping endothelial keratoplasty: The mechanics of iris buckling," Clinical and Experimental Ophthalmology 40(2), 182-186.

Lockington, D., Macdonald, E. C., Young, D., Stewart, P., Caslake, M. and Ramaesh, K. [2010] "Presence of free radicals in intracameral agents commonly used during cataract surgery," British Journal of Ophthalmology 94(12), 1674-1677.

Lorente, R., de Rojas, V., de Parga, P. V., Moreno, C., Varela, J., Landaluce, M. L., Méndez, J. and Lorente, B. [2012] "Intracameral phenylephrine 1.5\% for prophylaxis against intraoperative floppy iris syndrome: Prospective, randomized fellow eye study," Ophthalmology 119(10), 2053-2058.

Lundqvist, O., Koskela, T. and Behndig, A. [2014] "A paired comparison of intracameral mydriatics in refractive lens exchange surgery," Acta Ophthalmologica 92(5), 482485 .

Manvikar, S. and Allen, D. [2006] "Cataract surgery management in patients taking tamsulosin: Staged approach," Journal of Cataract and Refractive Surgery 32(10), 16111614 .

Miller, K. [2005] "Method of testing very soft biological tissues in compression," Journal of Biomechanics 38(1), 153-158.

Narendran, N., Jaycock, P., Johnston, R., Taylor, H., Adams, M., Tole, D., Asaria, R., Galloway, P. and Sparrow, J. [2009] "The cataract national dataset electronic multicentre audit of 55567 operations: Risk stratification for posterior capsule rupture and vitreous loss," Eye 23(1), 31.

Neff, K. D., Sandoval, H. P., de Castro, L. E. F., Nowacki, A. S., Vroman, D. T. and Solomon, K. D. [2009] "Factors associated with intraoperative floppy iris syndrome," Ophthalmology 116(4), 658-663.

Nguyen, D., Sebastian, R. and Kyle, G. [2007] "Surgeon's experiences of the intraoperative floppy iris syndrome in the United Kingdom," Eye 21(3), 443-444.

Sparrow, J. et al. [2011] "The cataract national data set electronic multi-centre audit of 55567 operations: Case-mix adjusted surgeon's outcomes for posterior capsule rupture," Eye 25(8), 1010.

Systémes, D. [2013] Abaqus 6.13 Analysis User's Guider, see also http://129.97.46. 200:2800/v6.13/.

Timoshenko, S. P. and Gere, J. M. [1961] Theory of Elastic Stability, McGrawHillKogakusha Ltd., Tokyo.

Townsend, P. R., Rose, R. M. and Radin, E. L. [1975] "Buckling studies of single human trabeculae," Journal of Biomechanics 8(3), 199-201.

Vazquez-Ferreiro, P., Carrera-Hueso, F. J., Barreiro-Rodriguez, L., Diaz-Rey, M., PoquetJornet, J. E., Ramón-Barrios, M. A. and Sanjuan-Cerveró, R. [2017] "Effectiveness of intracameral phenylephrine in achieving mydriasis and reducing complications during phacoemulsification: A systematic review and meta-analysis," Journal of Ocular Pharmacology and Therapeutics 33(10), 735-742.

Whitcomb, J. E., Barnett, V. A., Olsen, T. W. and Barocas, V. H. [2009] "Ex vivo porcine iris stiffening due to drug stimulation," Experimental Eye Research 89(4), 456-461.

Wittrick, W. H. and Williams, F. W. [1973] "An algorithm for computing critical buckling loads of elastic structures," Journal of Structural Mechanics 1(4), 497-518. 